\title{
Changes in the precipitation regime over the Italian Peninsula and their possible impacts on the electric system
}

\author{
R. Bonanno ${ }^{1}$ and P. Faggian ${ }^{1}$ \\ ${ }^{1}$ RSE S.p.A. - Ricerca sul Sistema Energetico, Milan, Italy \\ Received: 13-IV-2018 - Accepted: 30-VIII-2018 - Original version
}

Correspondence to: riccardo.bonanno@rse-web.it

\begin{abstract}
Extreme weather events represent serious risks for human activities and infrastructures. Hazards such as floods and droughts are one of the main challenges of the 21st century because of their significant societal and economic implications. In particular, their intensification puts a strain on the continuity of energy supply. The aim of this piece of work is to assess climate changes in average and extreme precipitations for the forthcoming decades over Italy. Two future scenarios have been elaborated (medium-term, 2021-2050 and long-term, 2071-2100) by analyzing MedCORDEX simulations at a horizontal resolution of about $50 \mathrm{~km}$ in two emission scenarios (RCP 4.5 and RCP 8.5). First, model values were bias-corrected by referring to E-OBS data, then a subset of standard indicators (WMO, 2009) was considered to investigate the change of climate signal. Despite some degree of uncertainty, the most significant climate change signals concern a general decrease in the average rainfall and an increase in extreme precipitation over some regions. The intensification of these events will lead to higher risks for electric failures; the reduction of mean precipitations will lead to a decline in hydroelectric power generation as well as water stress in cooling thermoelectric power plants.
\end{abstract}

Key words: heavy precipitation, droughts, regional climate models, energy system, climate change

\section{Introduction}

There is a general awareness that the climate is changing and extreme weather events (like storms, floods, heat waves and droughts) are steadily growing with increasing risks for the social, environmental and economic sectors.

The energy sector may also be very vulnerable to climate change. As mentioned by the U.S. Department of Energy (DOE) (2013), at least three major climate trends are relevant to the energy sector:

- Increasing air and water temperatures

- Decreasing water availability in some regions and seasons

- Increasing intensity and frequency of storm events, flooding, and rising sea level
In particular:

- Thermoelectric power generation facilities are at risk if water availability decreases and/or ambient air and water temperatures increase, as such meteorological conditions reduce the efficiency of cooling, increase the likelihood of exceeding water thermal intake as well as the effluent limits to protect local ecology, and increase the risk of partial or full shutdowns of generation facilities.

- Energy infrastructures sited along the coast are at risk from rising sea level, and storms with increasing intensity. In addition, oil and gas production, refining, and distribution, as well as electricity generation and distribution may be disrupted by higher storm surge and floods.

- Renewable energy resources, particularly hydropower, can be affected by changing precipitation patterns, 
increasing the frequency and intensity of droughts, and increasing temperatures.

- Electricity transmission and distribution systems may operate less efficiently when ambient air temperatures are higher, and they may face increasing risks of physical damage from more intense and frequent storm events or wildfires.

- Fuel transport is susceptible to increased interruption and delay if periods of drought and flooding, that affect water levels in rivers and ports, become more frequent.

- Electricity demand for cooling will likely increase in a warmer climate, while the demand for fuel oil and natural gas for heating is expected to decrease with increasing temperatures.

The Mediterranean region has been detected as one of the most prominent "Hot-Spots" in the future climate projections (Giorgi, 2006). Because of the interactions between a sub-tropical regime and mid-latitude synoptic activity driven by the westerly flow, small variations of the general circulation may shift mid-latitude storm tracks or sub-tropical highpressure cells, implying significant changes in the Mediterranean climate.

Many authors (Ababou et al., 2017; Longobardi and Villani, 2010; Arnone et al., 2013) report a strong negative trend observed in Mediterranean regions. The Italian Peninsula located at the center of the Mediterranean basin, may be affected by these changes, too (Coppola and Giorgi, 2010; Faggian and Giorgi, 2009).

As reported in the EU Report (2013), if left unmanaged, climate change may significantly affect the operational, financial, environmental and social performance of large fixed assets and infrastructures. Forzieri et al. (2016) raise serious concerns about an upsurge in climate hazard in Europe: the damages from climate extremes to critical infrastructures and key investments in the energy, transport, industrial and social sector, which at present total $€ 3.4$ billion a year, could triple by the 2020s, multiply six-fold by mid-century, and come to more than 10 times by the end of the century, with the highest economic losses projected for industry, followed by the energy sector. The severity of climate impacts on infrastructures may vary across the EU and according to individual locations and geophysical risk exposure.

The climate-weather conditions could be more serious again in the coming decades: many studies project a precipitation decrease over the Mediterranean area, especially during summer (Heinrich and Gobiet, 2011; Nikulin et al., 2011; Smiatek et al., 2009; Scoccimarro et al., 2015). These results are reinforced for Italy by some additional future scenarios gathered by analyzing different ENSEMBLES models (Faggian, 2015). Concerning this point, it is worth noting that the incidence of extreme precipitation in a warmer climate may possibly increase even in areas where the mean precipitation is projected to decrease (Allan and Soden, 2008; Trenberth et al., 2003).
Due to the complex topography and interactions between land and sea, the Mediterranean region is well known to be an area that is severely affected by extreme precipitation events (Alpert et al., 2002; Lionello et al., 2012). Substantial variability is found between the western and the eastern Mediterranean, but also on much smaller spatial scales due to the interaction of synoptic-scale and meso-scale processes triggering extreme precipitation (Doswell et al., 1998). Toreti et al. (2010), by studying extreme precipitation during the extended winter season at twenty Mediterranean coastal sites, note that at all the stations, precipitation extremes make a significant contribution (around 60\%) to seasonal totals.

The interaction between convective processes originating on the warm sea and sudden orographic lifting very close to the coast produces heavy rainfall. Often, the rain accumulated in $1 \mathrm{~h}$ accounts for the entire monthly average for that location (Rebora et al., 2012).

The undeniable intensification of extreme weather events also puts a strain on the continuity of the electricity service, as electricity systems were not designed to withstand many of the extreme weather events occurring today. Moreover, many parts of the electricity grid are old, outdated and in poor condition, making the systems even more vulnerable.

By the 2050s, so within the lifecycle of energy infrastructures, an increasing risk for network resilience and energy reliability is expected as climate change and extreme weather events could likely exceed the design parameters and other limits of power system assets and operations. Therefore, the need to assess the importance of climate change in order to help in the development of appropriate adaptation strategies is essential.

In the energy community, much of the attention has centered on the impact of water availability for the different processes of the energy sector and the energy sector's impact on water quality and quantity (Water-Energy Nexus). Both water and energy underpin economic and social development. As both water and energy needs are projected to increase, and the dependencies in both directions are set to intensify rapidly (IEA, 2016), it has become fundamental to understand the links between the two to anticipate future stress points and to implement policies, technologies and practices that soundly address the associated risks.

Precipitation is clearly a key variable in determining the water availability and can also represent a hazard for the energy sector in case of floods or drought conditions. In particular, in Italy the excess load of ice and snow on overhead power lines due to heavy wet snowfall events is the principal cause of "major power outages" of electric supply during the winter season (Bonelli et al., 2011), whereas in warm seasons strong thunderstorms, associated with hail, gusty winds, tornadoes and heavy rain may threaten electric supply, above all in regions between the Po Valley and the Alps. Some significant signals regarding precipitation changes over Italy have been pointed out by Brunetti et al. $(2004,2006)$ who found an increase of the intensity of precipitation and extreme events 
Table 1: List of Med-CORDEX models used in this study (www.medcordex.eu/index.php).

\begin{tabular}{|c|c|c|c|c|}
\hline RCM & GCM & Institute & Acronym & Resolution \\
\hline COSMO-CLM 4.8 & CMCC-CM & CMCC & CMCC CCLM & $\mathbf{0 . 4 4}^{\mathbf{0}}$ \\
\hline COSMO-CLM 4.8 & MPI-ESM-LR & GUF & $G U F C C L M$ & $\mathbf{0 . 4 4}^{\mathbf{}}$ \\
\hline Aladin 5.2 & CNRM-CM5 & CNRM & $A L A D I N$ & $\mathbf{0 . 4 4}^{\mathbf{o}}$ \\
\hline RegCM 4 & HadGEM2-ES & ICTP & $R E G C M$ & $\mathbf{0 . 4 4}^{\mathbf{o}}$ \\
\hline LMD & IPSL-CM5A-MR & IPSL & $L M D Z 4$ & $\mathbf{0 . 4 4}^{\mathbf{}}$ \\
\hline
\end{tabular}

in the last decades, despite a reduction in the number of wet days and the average annual precipitation.

The current climate information does not allow stakeholders and policy-makers to make accurate decisions on future infrastructure and operational requirements. Thus, the analysis of the state-of-art models represents the most appropriate tool to get new information about climate variability and change over the coming decades in order to improve vulnerability assessment and resilience planning.

The description of the regional distribution of increased extreme rain is not insignificant. As the attribution of precipitation variability to specific climate forcing is still uncertain, increasing the confidence of future projection of rainfall patterns remains a challenge (Sun et al., 2012).

Global climate models (GCMs) are powerful tools to assess global-scale climate variability and change: they generally operate at coarse horizontal resolutions (100-250 km) and are not yet appropriate for investigating the hydrological impacts of future extreme precipitation events at a local scale. The development of regional climate models (RCMs) led to a better representation of climate variability and change at local scales, and nowadays they are currently able to perform dynamical downscaling of GCMs at very high horizontal resolutions $(\sim 10 \mathrm{~km})$. The increased spatial resolution allows a better representation of surface heterogeneities and mesoscale atmospheric processes like convection (Fowler et al., 2007). In fact, the evaluation of the ability of RCMs to reproduce precipitation in the Mediterranean area in multimodel ensembles from the PRUDENCE (Jacob et al., 2007), ENSEMBLES (Lenderink and Van Meijgaard, 2010) and EURO-CORDEX (Jacob et al., 2014) projects has pointed out the improvement of the model's skills with an increase of the horizontal resolution (Rauscher et al., 2010).

The aim of this piece of work is to further investigate possible changes in mean and extreme precipitation regimes over the Italian Peninsula based on MedCORDEX models, which are the state-of-art regional simulations over the Mediterranean Basin. As part of the global CORDEX framework, the Med-CORDEX initiative (https://www.medcordex.eu/) provides regional climate projections for Europe at $50 \mathrm{~km}$ (EUR-44) and $12.5 \mathrm{~km}$ (EUR11) resolutions, complementing coarser regional simulations of former activities, like PRUDENCE and ENSEMBLES among others. These new models downscale the CMIP5 global climate projections (Taylor et al., 2012) in the new representative greenhouse gas concentration pathways (RCPs) (Moss et al., 2010; Detlef et al., 2011; Meinshausen et al., 2011). They use regional atmospheric, land surface, river and oceanic climate models and coupled regional climate system models to increase the reliability of regional climate information and understand the processes responsible for the Mediterranean climate variability and trends in order to give coherent climate scenarios for the coming decades with high spatial resolution. The paper is organized as follows: in Section 2 the data-sets and the methodology used are described; in Section 3 some future scenarios are discussed; in Section 4 the more meaningful conclusions are summarized.

\section{Data and methodology}

In this study, daily precipitation outputs from EUR44 models were used instead of EUR-11 because many more EUR-44 simulations were available compared to the higher resolution EUR-11. Ten simulations consisting of five Regional Climate Models (RCMs), nested in different Global Circulation Models (GCMs), forced by the RCP 4.5 and RCP 8.5 emission pathways, were considered (Table 1). Moreover, E-OBS (Haylock et al., 2008), a daily gridded observational dataset at $0.25^{\circ}$ resolution (about 25 $\mathrm{km}$ ), was used as reference data to characterize the current precipitation regimes.

It is known that regional climate models (RCMs) exhibit systematic biases in precipitation, due to limited process understanding or insufficient spatial resolution (Rauscher et al., 2010). Hence, RCM simulations need to be postprocessed (i.e. statistically adjusted, bias corrected) in order to produce reliable estimates of local scale climate used for climate impact assessments. Gudmundsson et al. (2012) reviewed different approaches using statistical transformations (classified into: distribution derived transformations, parametric transformations and nonparametric transformations) to postprocess RCM outputs and demonstrated an overall success in removing biases in RCMs. But they also demonstrated that the performances of methods differ substantially and stressed that each technique should be applied after checking its suitability for the data under consideration.

The approach adopted here is inspired by Piani et al. (2010), who use a parametric transformation, i.e. a gamma distribution-based quantile mapping technique for the biascorrection of daily precipitation. However, in our study, a nonparametric transformation, based on quantile-quantile 


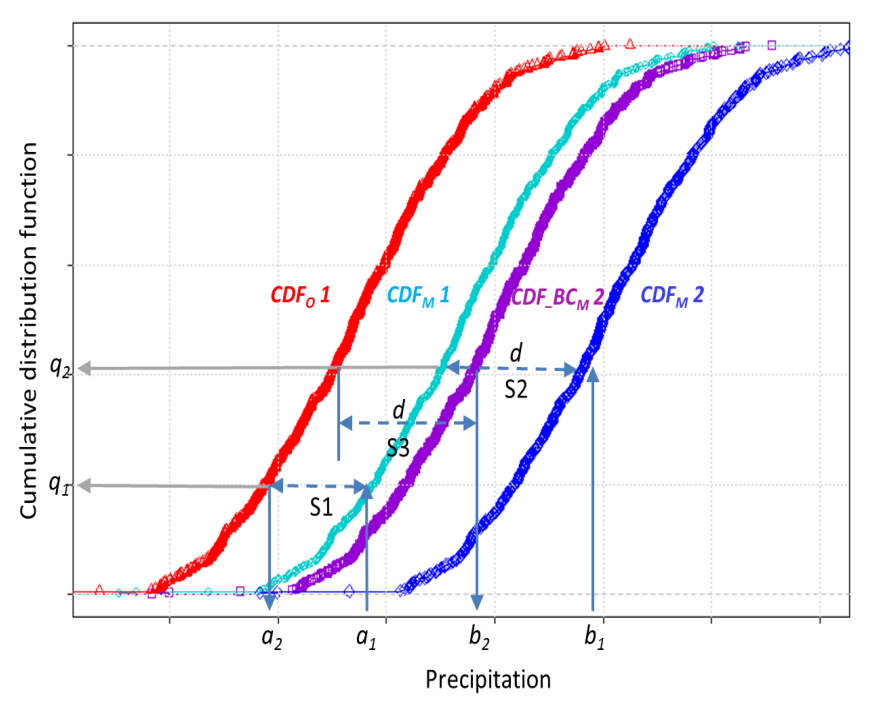

Figure 1: Schematic description of the Equidistant Quantile Mapping method (inspired by Sachindra et al. (2013)). $C D F_{O} 1$ represents the Cumulative Distribution Function $(C D F)$ of observed precipitations in the historical period; $C D F_{M} 1$ and $C D F_{M} 2$ the CDF of model precipitations in historical and future periods, respectively; $C D F_{-} B C_{M} 2$ is the analogue of $C D F_{M} 2$ but for model precipitations bias corrected with the Equidistant Quantile Mapping technique.

mapping (Boé et al., 2007), was chosen because it has the advantage of being independent from the underlying distribution of the variable considered. Moreover, the choice of a nonparametric bias-correction procedure was promoted by Gudmundsson et al. (2012) who showed clearly non-parametric transformations have the highest skill in systematically reducing biases in RCM precipitations compared to parametric ones.

Traditional quantile mapping is not recommended for bias correction. In fact, Cannon et al. (2015) found that quantile mapping may inflate relative trends in precipitation extreme indices, often by a substantial amount. Alternative methods, such as Equidistant Quantile Mapping ( $\mathrm{Li}$ et al., 2010) or Quantile Delta Mapping (Cannon et al., 2015) are preferred as they are able to preserve the changes in the quantiles simulated by climate models, considering that it is necessary to preserve the relative changes of variables related to atmospheric moisture, like precipitation, to maintain the physical scaling relationship with the model temperature changes. In this study, the Equidistant Quantile Mapping method (EQM) (Li et al., 2010; Sachindra et al., 2013) was used.

The thirty-year 1971-2000 period was analyzed as the reference period; the future scenarios were elaborated for two periods: 2021-2050 as the short-term scenario and 20712100 as the long-term one. The procedure adopted in this study consists of six steps:
1. First, the statistical consistency of the modelled precipitations with the climatology described by E-OBS was evaluated for each model in the reference period.

2. Then Med-CORDEX simulations were downscaled to E-OBS resolution by using bilinear interpolation.

3. The re-gridded fields were bias-corrected on the basis of E-OBS data in the reference period, by using a nonparametric transformation EQM ( $\mathrm{Li}$ et al., 2010; Sachindra et al., 2013). The procedure was applied over each grid point for each simulation and it is schematized in Fig. 1. The cumulative density function $(C D F)$ from E-OBS and RCM precipitation data were derived for the past climate (1971-2000) $\left(C D F_{O} 1\right.$ and $C D F_{M} 1$ respectively). For a given precipitation value $a_{1}$, the quantile value $q_{1}$ was obtained from $C D F_{M} 1$. From $C D F_{O} 1$, the precipitation value $a_{2}$ corresponding to $q_{1}$ was derived (S1). $\quad a_{2}$ is the corrected precipitation value of $a_{1}$. By repeating this procedure for all precipitation values, the mapping of $C D F_{M} 1$ onto $C D F_{O} 1$ was obtained and all the statistical properties of precipitation represented by $C D F_{M} 1$ were automatically matched with those of $C D F_{O} 1$. Then $\mathrm{CDFs}$ for the RCM precipitations were considered in the two future periods and in both RCPs. The difference between $C D F_{M} 2$ (future climate) and $C D F_{M} 1$ (past climate) was computed for each precipitation value (S2) and this difference was added to the corrected version of $C D F_{M} 1$ $\left(C D F_{-} B C_{M} 1\right.$ or $\left.C D F_{O} 1\right)(\mathrm{S} 3)$. The procedure was repeated until all the future precipitation values in $C D F_{M} 2$ had been corrected to obtain $C D F_{-} B C_{M} 2$ (eventual negative corrected precipitation values in biascorrect $C D F_{-} B C_{M} 2$ were set to zero).

4. A cross-validation was performed to test the effectiveness of applying the bias-correction method. To this end, a training period (consisting of all the evennumbered years in the historical period 1971-2000), and a validation period (consisting of all the odd-numbered years of the same time-framework) were considered. Then, the EQM technique was applied to the daily precipitation time series for each grid point in the training period and the resulting coefficients were used to perform the correction to precipitations in the validation years.

5. After verifying the effectiveness of the correction procedure, the whole set of RCM simulations were biascorrected.

6. To investigate the change of climate signal over Italy in the next decade, the indices PRCP DJF, PRCP MAM, PRCP JJA, PRCP SON, that represent winter, spring, summer and fall cumulated precipitations respectively, were computed. Moreover, the climate indicators defined by the Expert Team on Climate 


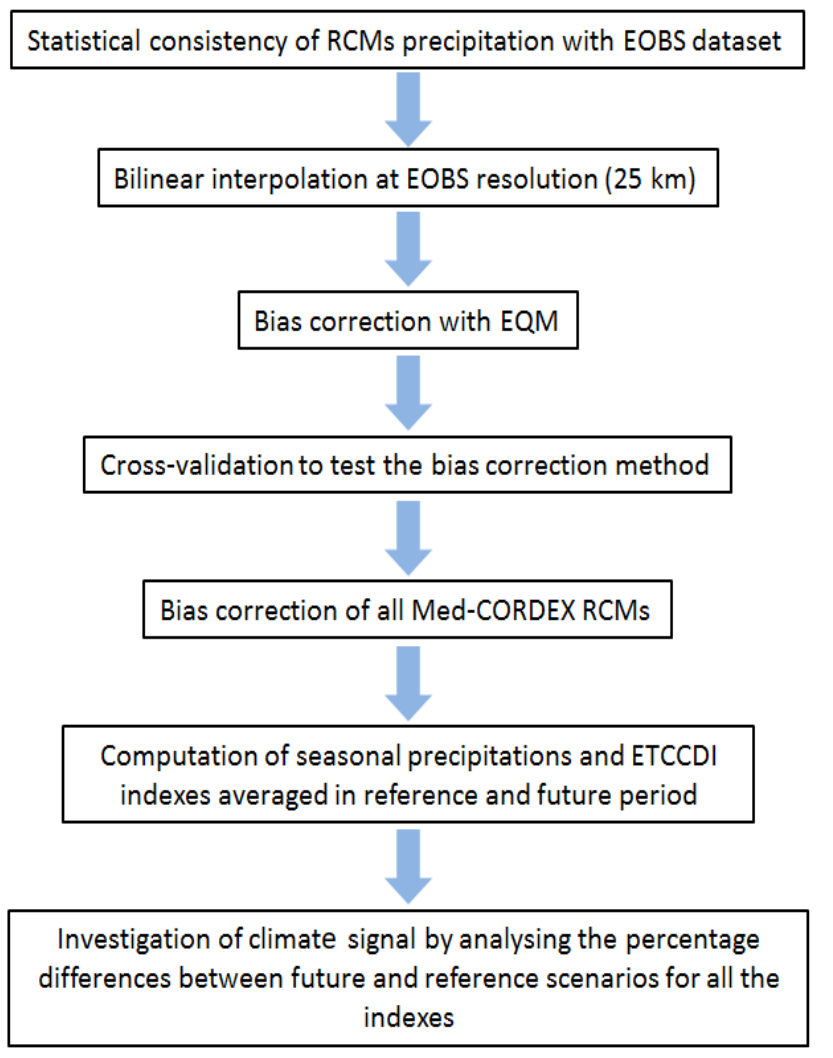

Figure 2: Flow chart representing the procedure adopted in this study.

Change Detection and Indices (ETCCDI) (WMO, 2009) related to the daily precipitations were considered. In fact, as such indices give redundant information, the investigation was focused on the three following indices:

- PRCPTOT (the total annual precipitation $[\mathrm{mm} / \mathrm{d}]$ ) to describe mean precipitation variations,

- R99PTOT (the annual total precipitation when daily wet day amount $>99$ th percentile $[\mathrm{mm} / \mathrm{d}])$ to investigate extreme events,

- CDD (the maximum number of consecutive dry days [days]) to get some information about droughts.

A schematic representation of the procedure adopted in this piece of work is shown in Fig. 2.

Before discussing the results, it may be useful to consider that the major drawback of all the statistical downscaling methods is that they rely on the assumption that the relationship between predictors and regional climate is unchanged in future climate conditions. However, this hypothesis cannot be totally verified. Moreover, two kinds of 'stationarity assumptions' underlie these approaches:
1. the physical parameterizations of RCMs remain applicable to climate change conditions;

2. the correction function established for the present climate is still applicable to altered climates.

It is also worth noting that the accuracy of the bias correction is limited by the quality of the observational data used. The E-OBS data has been found to be over compensated both in precipitation and temperature values, especially over the alpine regions, due to the limited number of meteorological stations used in spatial interpolation of highly complex orographic regions, leading to reduced areaaveraged data (Turco et al., 2013; Hofstra et al., 2009; Flaounas et al., 2012). Consequently, bias-corrected data may be less reliable in data-sparse regions.

\section{Discussion of the results}

\subsection{Historical period}

The Mediterranean Region is characterized by a typical seasonal cycle. Therefore, the performances of models have been verified on a seasonal scale (step 1).

Fig. 3 describes the climatology inferred from E-OBS (the first panel delimited by the green box) and MedCORDEX models (the other five panels, one for each model, in the same figure) through maps representing the mean seasonal precipitations over the 1971-2000 period: the winter (DJF), spring (MAM), summer (JJA) and fall (SON) fields are depicted clockwise, starting with the DJF map on the top-left corner of each panel.

By a visual comparison of each RCM and E-OBS panel, it is possible to see at a glance the skill of each model in describing the current precipitation regimes, according to E-OBS maps. It is remarkable that each model can capture the main futures of the precipitation, but often they show some biases, which may be more or less pronounced depending on the model, as well as the region and the season. However, as mentioned before, there are some deficiencies regards E-OBS dataset, especially in the Alpine regions, due to the limited number of meteorological stations used for characterizing this area. Thus, the results concerning this region are somewhat uncertain.

After downscaling the computing models through a bilinear interpolation (step 2) and applying a bias-correction to RCMs outputs (step 3), a cross-validation was performed (step 4). Table 2 reports the Mean Absolute Errors (MAE), compared to E-OBS data, calculated for each model in the validation period: the very low errors of the bias-corrected data (CORR) clearly highlight that they are more statistically coherent with the current climate than those that have not been bias-corrected (ORIG). Next, the bias correction was performed for all the RCM simulations (step 5). Further research was conducted by testing a seasonal bias correction, but without significant improvements compared to the annual correction (not shown). 

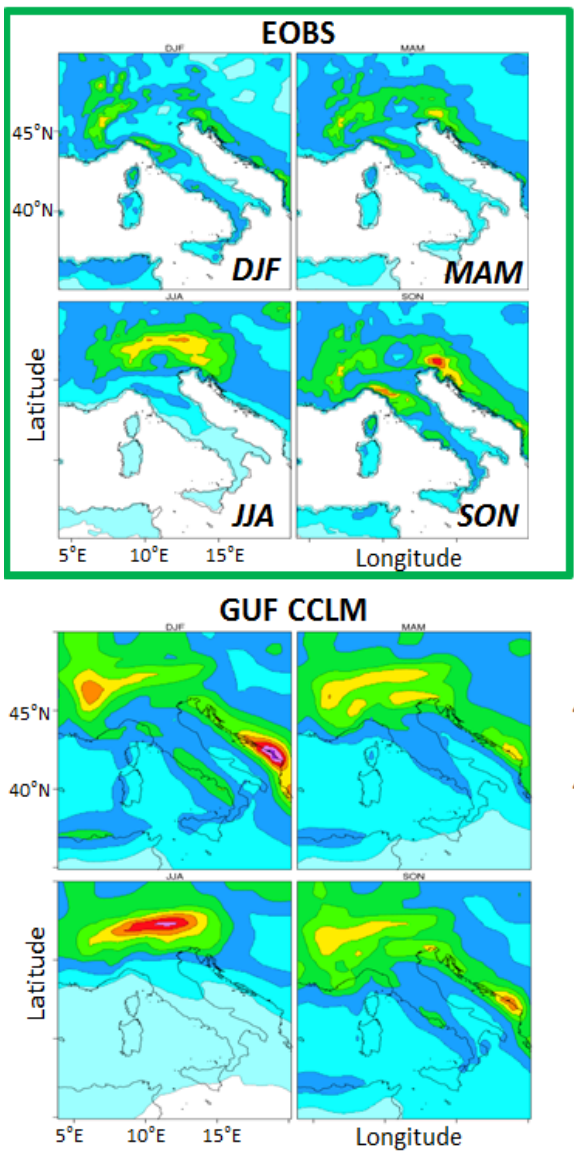

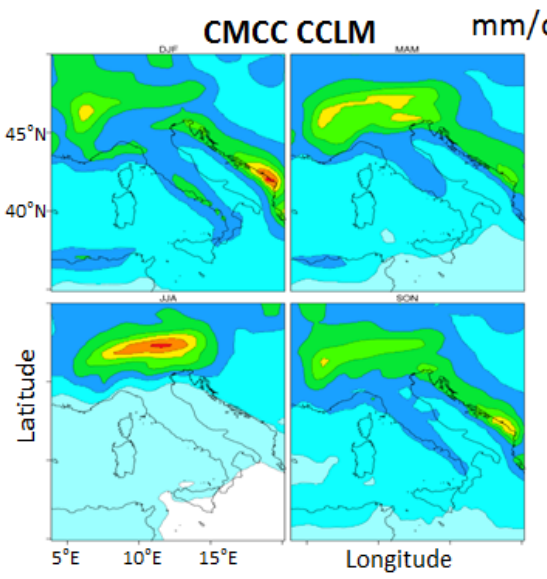

REGCM

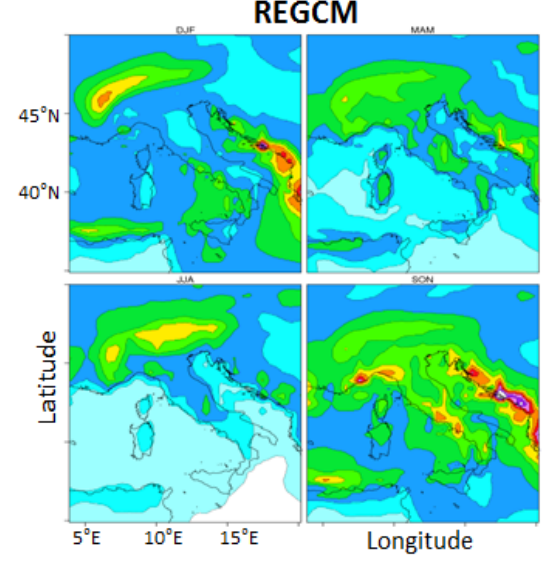

ALADIN
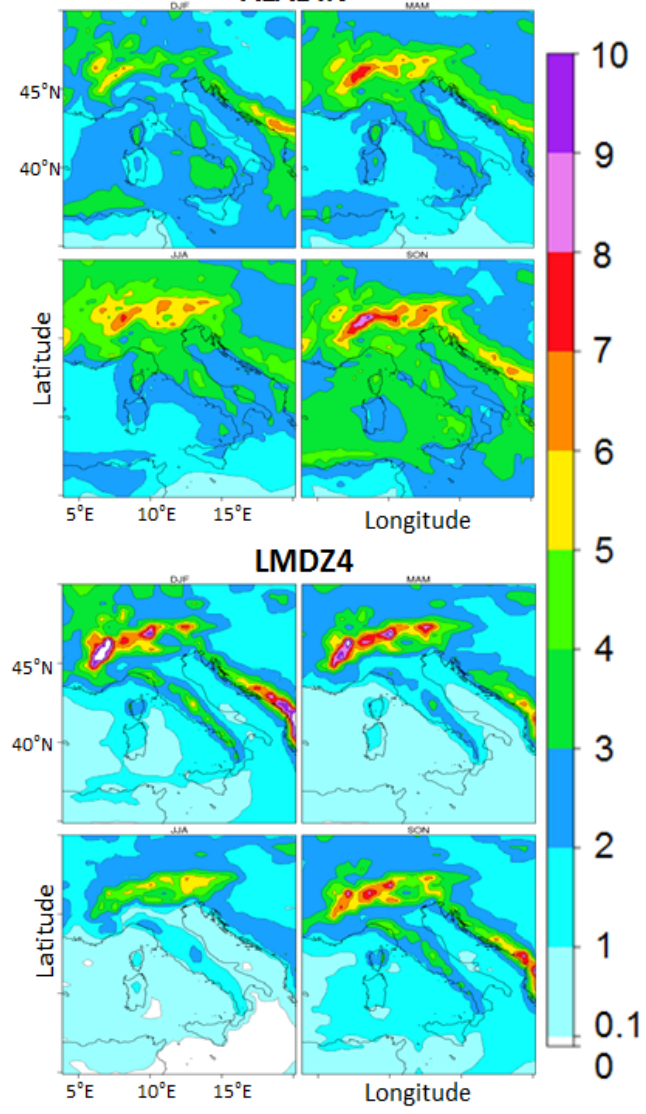

Figure 3: Seasonal precipitation maps for the 1971-2000 period for the reference dataset (E-OBS), inside the green box, and the five RCMs considered in this study.

Table 2: Seasonal values of Mean Absolute Error (MAE) computed for each model considered without bias correction (ORIG) and with bias correction (CORR).

\begin{tabular}{|c|c|c|c|c|c|c|c|c|c|c|}
\hline \multicolumn{10}{|c|}{ Mean Absolute Error (MAE) } \\
\hline & CMCC CLM & \multicolumn{2}{c|}{ ALADIN } & \multicolumn{2}{c|}{ GUF CLM } & \multicolumn{2}{c|}{ REGCM } & \multicolumn{2}{c|}{ LMDZ4 } \\
\hline & ORIG & CORR & ORIG & CORR & ORIG & CORR & ORIG & CORR & ORIG & CORR \\
\hline DJF & 0.08 & 0.07 & 0.10 & 0.06 & 0.10 & 0.05 & 0.10 & 0.08 & 0.12 & 0.10 \\
\hline MAM & 0.11 & 0.10 & 0.17 & 0.03 & 0.09 & 0.04 & 0.16 & 0.06 & 0.08 & 0.04 \\
\hline JJA & 0.07 & 0.06 & 0.24 & 0.09 & 0.06 & 0.06 & 0.13 & 0.06 & 0.08 & 0.07 \\
\hline SON & 0.10 & 0.04 & 0.17 & 0.08 & 0.10 & 0.04 & 0.19 & 0.04 & 0.10 & 0.07 \\
\hline
\end{tabular}

Lastly, climate change signals were investigated by calculating the aforementioned indices from each model. The analysis was done for each model and by considering the whole set of simulations, i.e. without neglecting the coincidence between models to test the confidence of the results (step 6).

\subsection{Future scenarios}

From this point on, the percentage variations of the indices PRCPTOT, R99PTOT and CDD regarding the reference period (1971-2000) are considered separately. Fig.
4 to 7 show the results for the two future periods (short-term and long-term) and under the two forcings: RCP 4.5 and RCP 8.5 .

Each figure consists of different maps reporting the percentage variations of the three indices (one for each row), for each model (one for each column): yellow, orange and reddish colors are used to express a decrease in the index; on the contrary, light blue, cyan, blue and violet colors are used to indicate an increase of it. The black dots mark the pixels for which the difference between the future scenario and historical period is statistically significant at $5 \%$ according to the Wilcoxon rank-sum test. 


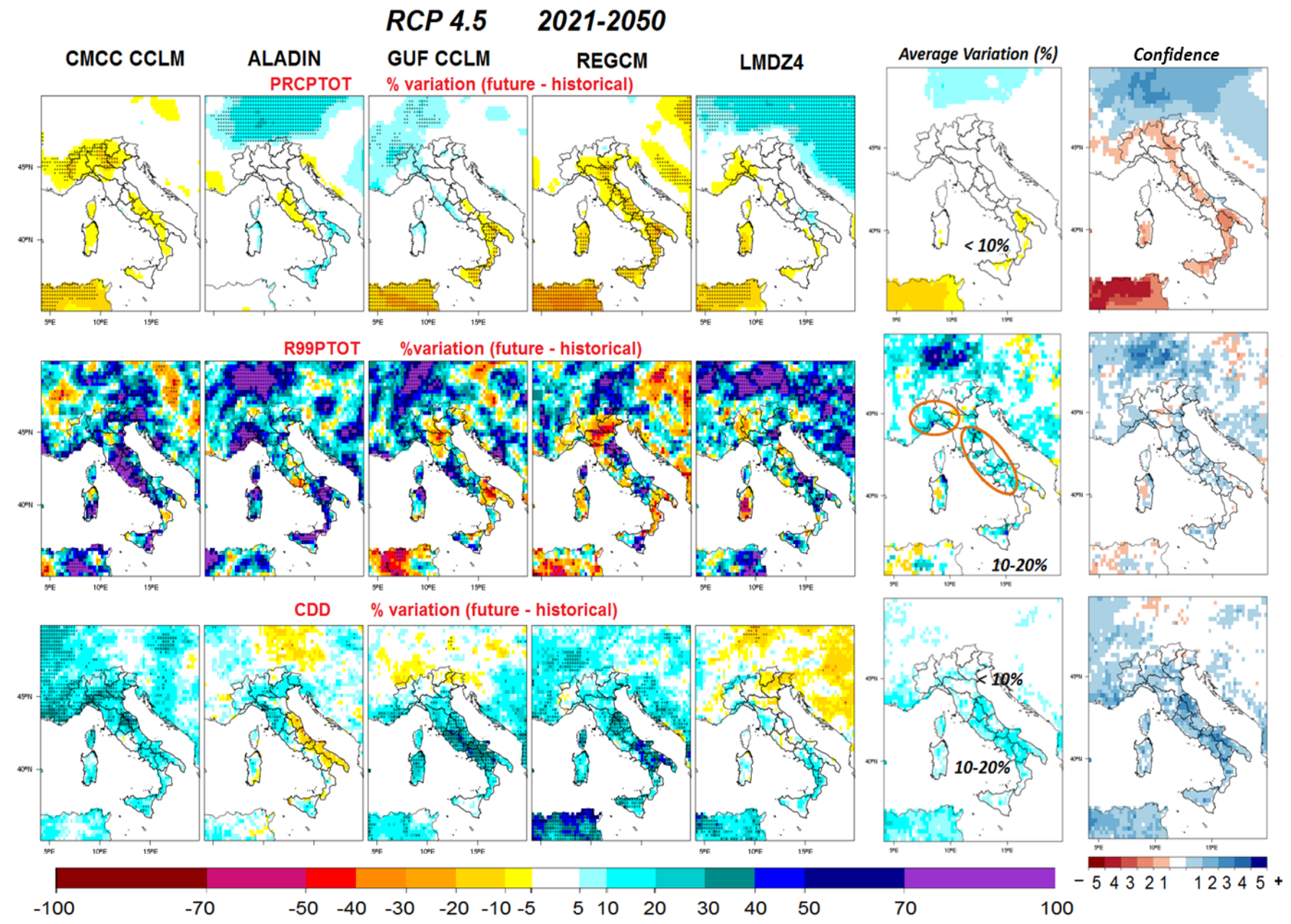

Figure 4: Maps of percentage variations of the indices PRCPTOT, R99PTOT and CDD in the short-term 2021-2050 period compared to the historical 1971-2000 period in RCP 4.5 configuration. The corresponding Average Variation and Confidence maps are reported on the right.

On the right of each row, two additional panels are reported: "Average variation" and "Confidence" maps: the first map is inferred by considering only the models giving a statistically significant signal and is visualized using the same colors as for describing the percentage variations of the single models; the second one states the coincidence between the models (i.e. the confidence of the results) about the sign of changes projected for that index. This map is obtained by counting the models giving a statistically significant increase/decrease and it is visualized according its corresponding legend. The pixels are white where there are no statistically significant signals or the number of models that indicate a growing trend is equal to that of models that indicate the opposite. If the models disagree in the projection, the corresponding pixels are depicted with a color corresponding to the stronger signal, whereas the small dot inside the same pixel is colored according the weaker signal.

\subsubsection{Future projections under RCP 4.5 forcing in the} short-term period (Fig. 4)

By analyzing the maps relating PRCPTOT changes (first row of the figure), some models show a significant decrease $(\sim 10-20 \%)$ of the total precipitation in the 20212050 period, especially in the south, with low-medium confidence (1-2 models out of 5) in Southern Italy; elsewhere generally no information or low confidence about future decrease has been found.

As far as the R99PTOT index (second row of figures) is concerned, a strong increase in extreme precipitation is inferred, some areas are even projected to be affected by an increase of about $70-100 \%$. However, because of the high spatial variability (different patterns are inferred from the models), there is very low confidence in localizing the increase of this index.

Regarding CDD, the projections give an increase of between 20 and $40 \%$. In particular, a likely increase of CDD 


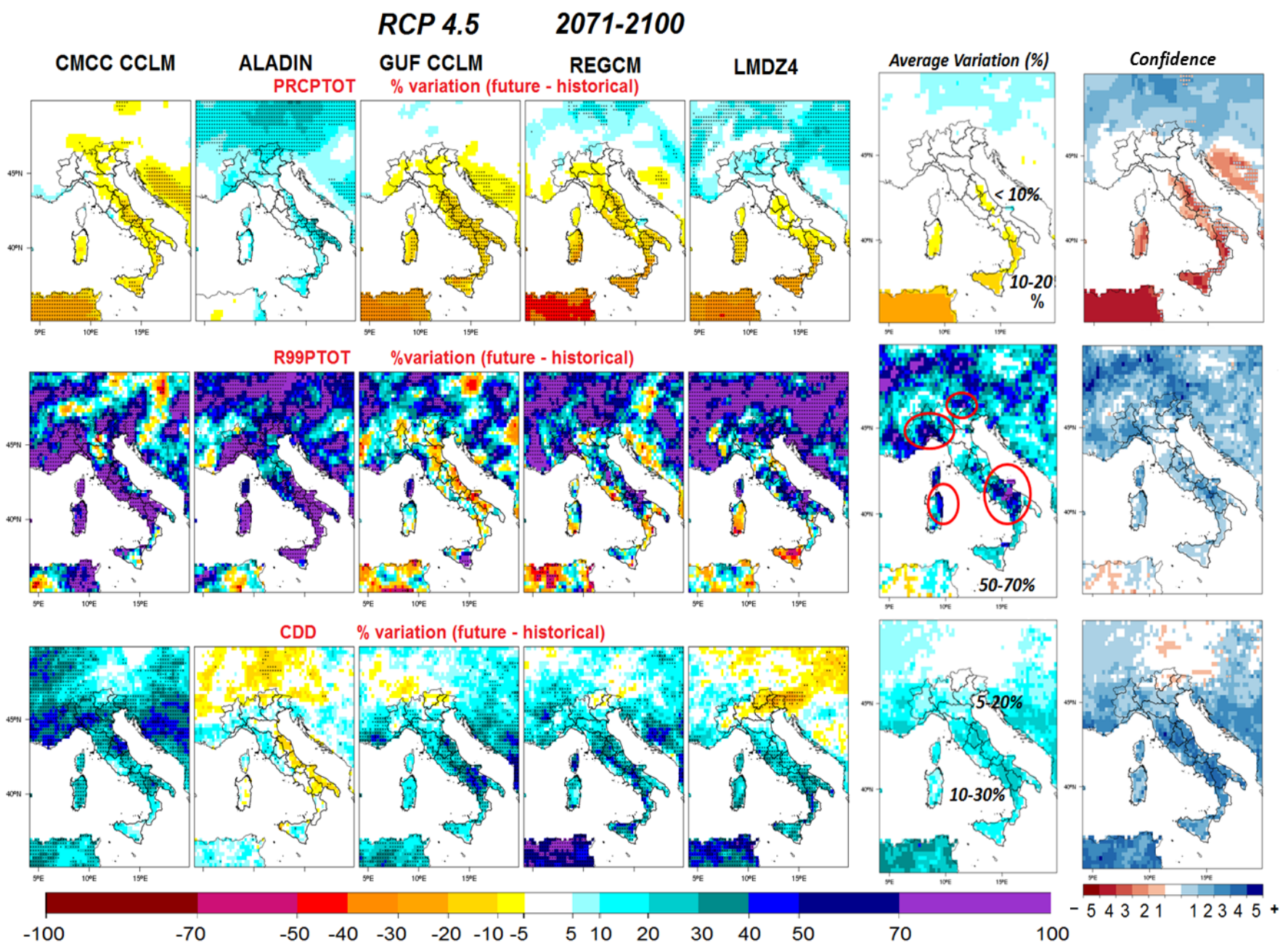

Figure 5: As for Fig. 4, but for the long-term 2071-2100 scenario.

in Central and Southern Italy (2-3 models out of 5) can be noticed.

\subsubsection{Future projections under RCP 4.5 forcing in the long- term period (Fig. 5)}

The models describe a general decrease in PRCTOT of between 20 and $40 \%$ by 2071-2100, with the exception of ALADIN that shows a statistically significant increase over the North Eastern Alpine Region and the east coasts of Southern Italy. The results of the decrease in total precipitation in Central and Southern Italy have to be considered with medium confidence (2-3 models out of 5) over the Apennines and Sardinia. A high coincidence among models (3-4 models out of 5) is found locally in some regions in Southern Italy (Calabria and Sicily). Elsewhere, generally no information or low confidence in precipitation changes was found (in the North-Eastern Alpine Regions, namely Trentino Alto Adige).

The R99PTOT scenario is depicted more clearly for the long-term than the short-term, as a stronger increase in frequency and intensity of extreme precipitations is projected. By considering the statistically significant changes together, a likely increase (2-3 models out of 5) comes up locally in north eastern, north western Italy and over the Apennines between Central and Southern Italy.

A strong increase of CDD is expected with statistically significant changes of between 30 and 50\%. As described by the confidence map, there is a high coincidence of such results (3-4 models out of 5) and, therefore, mediumhigh confidence about the increase of consecutive dry days over Central and Southern Italy; instead, a low-medium coincidence between models (1-2 models out of 5) is found for Northern Italy.

\subsubsection{Future projections under RCP 8.5 forcing in the} short-term period (Fig. 6)

The short-term RCP 8.5 scenarios are very similar to the short-term RCP 4.5 ones, despite some small differences. A decrease in PRCPTOT with low-medium confidence (2/3 models out of 5$)$ is projected over the southernmost 


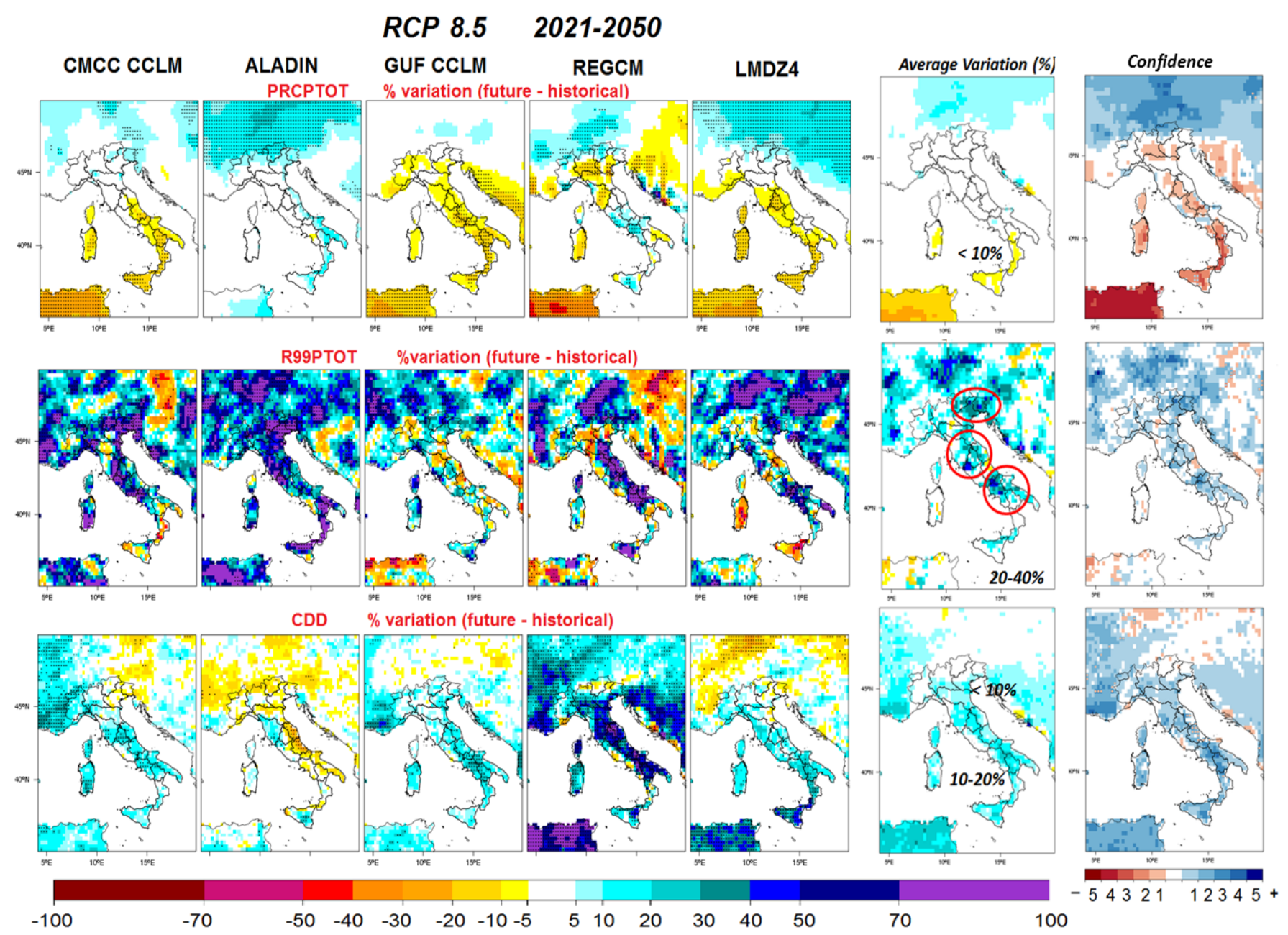

Figure 6: As for Fig. 4 but for simulations in RCP 8.5 configuration.

part of Italy (Calabria and Sicily regions) and Sardinia; a precipitation increasing with low-medium confidence is inferred for northernmost Italy (Trentino Alto Adige), whereas in the rest of Italy the signal is weaker and there are some uncertainties about the precipitation decrease (1 model out of 5).

A strong intensification of extreme events is expected locally, because R99PTOT is projected to increase by about $70-100 \%$ over some areas. Despite the high spatial variability of this index, clearer results are gathered in comparison with RCP4.5 ones, as there is medium confidence about the increase of extreme events over the North-East and a few regions in Central Italy. Elsewhere, no signals are appreciable because of the absence of significant trends.

All the models describe an increase in the length of dry spells, analogously with the RCP 4.5 projections, except for REGCM, the results of which show appreciable increases of CDD (generally between 50 and $70 \%$ with some peaks in Central Italy of up to 70\%). The GUF-CLM model shows a weaker increase compared to the other scenarios in the same period, whereas ALADIN shows a stronger and statistically significant decrease in CDD index on the Adriatic coast in Central Italy. As a result, some uncertainties characterize the expected increase of CDD by 2021-2050 in RCP 8.5 scenario, projected by 2-3 models over southern areas, 12 models over Central Italy and at least one model for the North.

\subsubsection{Future projections under RCP 8.5 forcing in the long- term period (Fig. 7)}

Under the RCP 8.5 emission scenario in the long-term 2071-2100 period, all the models project a general decrease in PRCPTOT of between $20-40 \%$, locally of up to $50 \%$ in Southern Italy and Sardinia, with the only exception of ALADIN model whose index increases significantly in the North Eastern Alpine Regions and on the East Adriatic coast of Central and Southern Italy. As reported by the confidence map, total precipitation is expected to decrease over Central Italy (especially over the Apennines) and Southern Italy with reasonably high confidence (4 models out of 5 with 


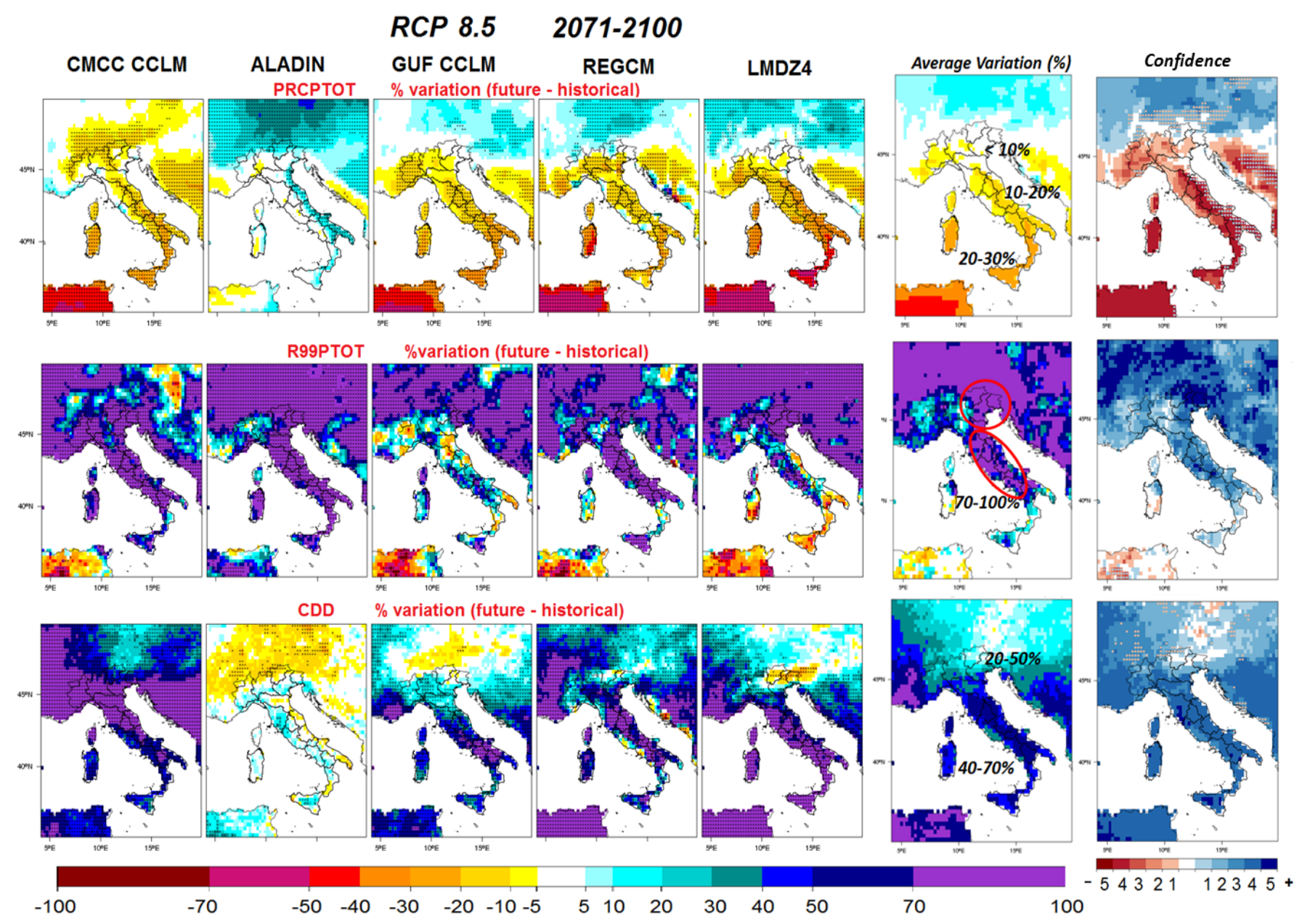

Figure 7: As for Fig. 4 but for the long-term scenario in RCP 8.5 configuration.

the ALADIN's evident countertrend on the east Adriatic coast), whereas a precipitation decrease along the Central Tyrrhenian coast and in Northern Italy is projected with low-medium confidence.

The R99PTOT index points out a stronger increase in frequency and intensity of extreme events by the end of the century, compared to the RCP 8.5 short-term projections and the RCP 4.5 scenarios. In particular, according to some models (CNMM-CCLM, ALADIN and REGCM), most of our country is projected to be affected by a statistically significant increase $(70-100 \%)$ of heavy precipitations: a very likely increase (3-4 models out of 5) of extreme precipitation is projected over most of central Italy and locally in the south, as well as the Northern Alps. A virtually certain increase of extreme events is expected in North Eastern Italy (Trentino Alto Adige, Friuli Venezia Giulia and Veneto regions). Elsewhere, a low-medium likely increase of extremes was found.

The models point out a remarkable increase (of at least $20 \%$ ) in the length of dry spells over most of Italy, except for ALADIN simulations whose CDD values are expected to decrease over the Alps without significant signals elsewhere.
Also, the LMDZ4 model projects CDD decreasing over the Alpine region, but only for the eastern part. On the whole, an increase in the length of dry spells is likely or very likely (more than 60\%) over most of Italy at the end of the century, except for the Alps, above all in the eastern part for which there is a lower model coincidence.

Considering the analysis from a seasonal point of view (the maps are not shown for the sake of brevity), a likely decrease in precipitation is expected to be more pronounced in the summer season: for RCP 4.5, $10 \%$ in the short term and $10-20 \%$, locally up to $20 \%$, in the long term; for RCP 8.5 , on average $\sim 10-20 \%$ in short term and $\sim 40-50 \%$ in the long term. In this last case, with high confidence (4 models out of 5), Central Italy and Sardinia will be affected by the most serious precipitation decrease. In the spring and fall seasons, the magnitude and the confidence of decrease are generally lower. Instead, in the winter season, an increase of precipitation is likely over Northern Italy, especially over the Alps with confidence ranging from medium to high degree for long-term scenarios under RCP 8.5 forcing. 


\section{Summary}

This study assesses the changes in precipitation regimes expected over Italy, considering their significant implications for both energy and water security. Some future projections (short- and long-term) regarding the changes of precipitation regimes expected over Italy have been elaborated through the computation of some ETCCDI indices. By focusing the analysis of three of them (PRCPTOT, R99PTOT and CDD) on the two emission scenarios RCP 4.5 and RCP 8.5, some significant signals have been found, such as the "Average variation" and "Confidence" maps that Fig. 4 to 7 show:

- PRCPTOT maps highlight a weak signal of precipitation decrease in the short term in both the RCP forcings over southern areas (generally lower than 10\%) with medium-low confidence. In the long-term RCP 8.5 configuration, a very likely decrease in total precipitation is expected in Central and Southern Italy and Sardinia (20$30 \%$ ), whereas a lower decrease is projected for Central Italy (10-20\%), 10\% lower than for elsewhere. The long-term RCP 4.5 projection has an analogous pattern but with lower confidence with decreasing values between 10 and $20 \%$ in the southernmost regions, $10 \%$ lower than for elsewhere.

- Concerning the R99PTOT changes, an increase in extreme precipitation was found by the middle of the century with some uncertainties under RCP 4.5 forcing, whereas the signal is a bit clearer (low-medium confidence) under RCP 8.5 hypothesis over Northeast and Central Italy, with an increase of about 30-40\%, locally exceeding $40 \%$ for the regions characterized by the highest occurrence probabilities. In the long term, the results are: in RCP 8.5 forcing, the risk for an increase of strong convective phenomena and floods affects the northeast, northwest and center of the Italian Peninsula with medium confidence, it is almost certain for northeast areas where the increase of R99PTOT is expected to be between 70 and 100\%; in RCP 4.5 configurations, an increase of extreme precipitations is projected with low-medium confidence above all in the northwest areas, the northeastern Alpine regions (mainly Trentino Alto Adige) and in the regions between Central and Southern Italy, with variations of between 50 and $70 \%$, locally also up to $70 \%$.

- In the short term, all the scenarios highlight an increase in the length of dry spells with low-medium confidence for Central and Southern Italy and low confidence in the North. In the regions where the highest coincidence among the models is found, the CDD variations ranges from 5 to 20\%. By 2017-2100 an increase of CDD is expected. The variation is about $10-30 \%$ with mediumhigh confidence in Central and Southern Italy in RCP 4.5 configuration and ranges between 40 and $70 \%$ with very high confidence (even though lower over the Alps) under RCP 8.5 forcing.
To summarize the results, most models coincide in projecting a general decrease in total precipitation (more likely in Central and Southern Italy), as well as an increase in heavy precipitations events and a lengthening of dry periods, even if sometimes the signal of change is not statistically significant and some difficulties were found in localizing the projections.

In spite of some uncertainties and considering that the high variability in projected geographical patterns of heavy precipitation intensity is due to structural and parametric model uncertainty and internal climate variability (e.g. Knutti and Sedlácek (2013); Schindelr et al. (2015), the scenarios highlight increasing risks for the electroenergy system, above all, under the RCP 8.5 configuration describing very high greenhouse gas emission; the reduction of precipitations and the lengthening of dry spells may hinder hydroelectric power generation and threaten water resource for cooling the thermoelectric power plants. Extreme events may cause a decrease in hydroelectric power generation, too, as flood events may hamper the management of hydroelectric plants. More generally, an increase of extreme events may intensify local flooding (e.g. flash flooding and urban flooding) and lead to damages to electric infrastructures with further risks for electric failures.

Moreover, combined with a warmer climate, fewer precipitations and longer drought periods may lead to an increase in energy power demand and also to a loss of capacity in transporting energy.

There is an urgent need to adapt to climate change, even because such phenomena are also correlated to heavy wet snowfalls in winter and deep convection with strong wind gusts and hail in the other seasons.

A new high-resolution dataset of climate simulations coming from the Euro-CORDEX project is available for impact studies. At the moment, this dataset consists of 14 models at $12 \mathrm{~km}$ resolution, both for the RCP 8.5 scenario (2006-2100) and for the historic period (1961-2005).

By using these data, further investigation is planned in order to get more reliable information, thanks to the much higher number of climate models available. A detailed analysis for the temperature is also planned to have a more complete view of the combined effects of extreme temperatures and precipitations on the electro-energy system and, therefore, to gather information required to develop appropriate resilience solutions. All this information will be used to quantify the impacts mentioned above on the electroenergy system.

Acknowledgements. This piece of work was financed by the Research Fund for the Italian Electrical System under the Contract Agreement between RSE S.p.A. and the Italian Ministry of Economic Development - General Directorate for Nuclear Energy, Renewable Energy and Energy Efficiency, stipulated on July 29, 2009, in compliance with the Decree of November 11, 2012. We would like to acknowledge the World Climate Research Programme's Working Group on Regional Climate, and the Working Group on Coupled Modelling, former coordinating body of CORDEX and the panel responsible for CMIP5. We would also like to acknowledge the E-OBS (Haylock et al., 2008) dataset from the EU-FP6 project ENSEMBLES (http://ensembleseu.metoffice.com) and the data providers for the ECAD project (http://www.ecad.eu). 


\section{References}

Ababou, A., Bouthiba, A., Chouieb, M., and Reguieg Yssaad, H., 2017: Drought assessment and rainfall trend analysis in a southern Mediterranean watershed during the last century (1914-2011), Tethys, 14, 10-22, doi:10.3369/tethys.2017.14.02.

Allan, R. and Soden, B., 2008: Atmospheric warming and the amplification of precipitation extremes, Science, 321, 14811484.

Alpert, P., Ben-Gai, T., Baharad, A., Benjamini, Y., Yekutieli, D., Colacino, M., Diodato, L., Ramis, C., Homar, V., and Romero, R. e. a., 2002: The paradoxical increase of Mediterranean extreme daily rainfall in spite of decrease in total values, Geophys. Res. Lett., 29, 31-1.

Arnone, E., Pumo, D., Viola, F., Noto, L., and La Loggia, G., 2013: Rainfall statistics changes in Sicily, Hydrol. Earth Syst. Sci., 17.

Boé, J., Terray, L., Habets, F., and Martin, E., 2007: Statistical and dynamical downscaling of the Seine basin climate for hydro-meteorological studies, Int. J. Climatol., 27, 1643-1655, doi:doi:10.1002/joc.1602.

Bonelli, P., Lacavalla, M., Marcacci, P., Mariani, G., and Stella, G., 2011: Wet snow hazard for power lines: a forecast and alert system applied in Italy, Nat. Hazards Earth Syst. Sci., 11, 2419-2431, doi:10.5194/nhess-11-2419-2011.

Brooks, H., 2013: Severe thunderstorms and climate change, Atmos. Res., 123, 129-138, doi:10.1016/j.atmosres.2012.04.002.

Brunetti, M., Maugeri, M., Monti, F., and Nanni, T., 2004: Changes in daily precipitation frequency and distribution in Italy over the last 120 years, J. Geophys. Res., 109, doi: 10.1029/2003JD004296.

Brunetti, M., Maugeri, M., Monti, F., and Nanni, T., 2006: Temperature and precipitation variability in Italy in the last two centuries from homogenised instrumental time series, Int. J. Climatol., 26, 345-381.

Cannon, A., Sobie, S., and Murdock, T., 2015: Bias correction of GCM precipitation by quantile mapping: How well do methods preserve changes in quantiles and extremes?, J. CLIMATE, 28, 6938-6959, doi: 10.1175/JCLI-D-14-00754.1.

Coppola, E. and Giorgi, F., 2010: An Assessment of Temperature and Precipitation Change Projections over Italy from Recent Global and Regional Climate Model Simulation, Int. J. of Climatology, 30, 11-32.

Detlef, P. v. V., Edmonds, J., Kainuma, M., Riahi, K., Thomson, A., Hibbard, K., Hurtt, G., Kram, T., Krey, V., Lamarque, J., Masui, T., Meinshausen, M., Nakicenovic, N., Smith, S., and Rose, S., 2011: The representative concentration pathways: an overview, Clim. Change, 109, 5-31, doi: 10.1007/s10584-011-0148-z.

Doswell, C. A., Ramis, C., Romero, R., and Alonso, S., 1998: A diagnostic study of three heavy precipitation episodes in the western Mediterranean region, Weather Forecast., 13, 102-124.

European Union Comission staff working document, 2013: Adapting infrastructure to climate change, SWD (2013) 137 final.

Faggian, P., 2015: Climate Change Projections for Mediterranean Region with Focus over Alpine Region and Italy, J. Environ. Sci. Eng, doi:10.17265/2162-5263/2015.09.004.

Faggian, P. and Giorgi, F., 2009: An Analysis of Global Model Projections over Italy, with Particular Attention to the Italian Greater Alpine Region (GAR), Clim. Change, 96, 239-258.

Flaounas, E., Drobinski, P.and Borga, M., Calvet, J., Delrieu, G., Morin, E., Tartari, G., and Toffolon, R., 2012: Assessment of
Gridded Observations Used for Climate Model Validation in the Mediterranean Region: the HyMeX and MED-CORDEX Framework, Environ. Res. Lett., 7, doi:10.1088/1748-9326/7/2/02401.

Forzieri, G., Bianchi, A., Herrera, M., Batista e Silva, F., Lavalle, C., and Feyen, L., 2016: Resilience of large investments and critical infrastructures in Europe to climate change, JRC Report.

Fowler, H. J., Ekström, M., Blenkinsop, S., and Smith, A. P., 2007: Estimating change in extreme European precipitation using a multimodel ensemble, J. Geophys. Res., 112, d18104.

Giorgi, F., 2006: Climate Change Hot-Spots, Geophys. Res. Lett., 33, L08 707.

Gudmundsson, L., Bremnes, J., Haugen, J., and Engen-Skaugen, T., 2012: Downscaling RCM precipitation to the station scale using statistical transformations - a comparison of methods, Hydrol. Earth Syst. Sci., 16, 3383-3390, doi:10.5194/hess-163383-2012.

Haylock, M., Hofstra, N., Klein Tank, A., Klok, E., Jones, P., and New, N., 2008: A European daily high-resolution gridded data set of surface temperature and precipitation for 1950-2006, J. Geophys. Res (Atmospheres), 113, D20 119, doi:10.1029/2008JD10201.

Heinrich, G. and Gobiet, A., 2011: The future of dry and wet spells in Europe: A comprehensive study based on the ENSEMBLES regional climate models, Int. J. Climatol., 32, 1951-1970.

Hofstra, N., Haylock, M., New, M., and Jones, P., 2009: Testing E-OBS European High-Resolution Gridded Data Set of Daily Precipitation and Surface Temperature, J. Geophys. Res., 114, D21 101, doi:10.102972009JD11799.

International Energy Agency, 2016: Water Energy Nexus, www.iea.org.

Jacob, D., Bärring, L., Christensen, O. B., Christensen, J. H., de Castro, M., Deque, M., Giorgi, F., Hagemann, S., Hirschi, M., Jones, R., Kjellström, E., Lenderink, G., Rockel, B., Sánchez, E., Schär, C., Seneviratne, S. I., Somot, S., van Ulden, A., and van den Hurk, B., 2007: An inter-comparison of regional climate models for Europe: model performance in present-day climate, Clim. Change, 81, 31-52, doi:10.1007/s10584-006-9213-4.

Jacob, D., Petersen, J., Eggert, B., Alias, A., Christensen, O. B., Bouwer, L. M., Braun, A., Colette, A., Déqué, M., Georgievski, G., Georgopoulou, E., Gobiet, A., Menut, L., Nikulin, G., Haensler, A., Hempelmann, N., Jones, C., Keuler, K., Kovats, S., Kröner, N., Kotlarski, S., Kriegsmann, A., Martin, E., van Meijgaard, E., Moseley, C., Pfeifer, S., Preuschmann, S., Radermacher, C., Radtke, K., Rechid, D., Rounsevell, M., Samuelsson, P., Somot, S., Soussana, J.-F., Teichmann, C., Valentini, R., Vautard, R., Weber, B., and Yiou, P., 2014: EUROCORDEX: new high-resolution climate change projections for European impact research, Reg. Environ. Change, 14, 563-578.

Knutti, R. and Sedlácek, J., 2013: Robustness and uncertainties in the new CMIP5 climate model projections, Nature Climate Change, 3, 369-373, doi:10.1038/nclimate1716.

Lenderink, G. and Van Meijgaard, E., 2010: Linking increases in hourly precipitation extremes to atmospheric temperature and moisture changes, Environ. Res. Lett., 5, 025208.

Li, H., Sheffield, J., and Wood, E., 2010: Bias correction of monthly precipitation and temperature fields from Intergovernmental Panel on Climate Change AR4 models using equidistant quantile matching, J. Geophys. Res., 115, D10 101, doi: 10.1029/2009JD012882. 
Lionello, P., Abrantes, F., Congedi, L., Dulac, F., Gacic, M., Gomis, D., Goodess, C., Hoff, H., Kutiel, H., Luterbacher, J., Planton, S., Reale, M., Schröeder, K., Struglia, V., Toreti, A., Tsimplis, M., Ulbrich, U., and Xoplaki, E., 2012: Mediterranean Climate Background Information. In: Lionello, P. (ed) The climate of the Mediterranean region: from the past to the future, Elsevier, pp. 266-290.

Longobardi, A. and Villani, P., 2010: Trend analysis of annual and seasonal rainfall time series in the Mediterranean area, Int. J. Climatol., 30, 1538-1546, doi: 10.1002/joc.2001.

Meinshausen, M., Smith, S., Calvin, K., Daniel, J., Kainuma, M., Lamarque, J.-F., Matsumoto, K., Montzka, S., Raper, S., Riahi, K., Thomson, A., Velders, G., and van Vuuren, D., 2011: The $R C P$ greenhouse gas: concentrations and their extensions from 1765 to 2300, Clim. Change, 35, 213-241, doi:10.1007/s10584011-0156-z.

Moss, R., Edmonds, J., Hibbard, K., Manning, M., Rose, S., van Vuuren, D., Carter, T., Emori, S., Kainuma, M., Kram, T., Meehl, G., Mitchell, J., Nakicenovic, N., Riahi, K., Smith, S., Stouffer, R., Thomson, A., Weyant, J., and Wilbanks, T., 2010: The next generation of scenarios for climate change research and assessment, Nature, 463, 747-756, doi:10.1038/nature08823.

National Renewable Energy Laboratory (NREL), U.S. Department of Energy, 2013: Energy Sector Vulnerabilities to Climate Change and Extreme Weather, U.S. Department of Energy, available at: http://energy.gov/sites/prod/files/2013/07/f2/20130710Energy-Sector-Vulnerabilities-Report.pdf.

Nikulin, G., Kjellstrom, E., Hansson, U., Strandberg, G., and Ullerstig, A., 2011: Evaluation and future projections of temperature, precipitation and wind extremes over Europe in an ensemble of regional climate simulations, Tellus, 63A, 41-55, doi:10.1111/j.1600-0870.2010.00466.x.

Piani, C., Haerter, J. O., and Coppola, E., 2010: Statistical bias correction for daily precipitation in regional climate models over Europe, THEOR APPL CLIMATOL, 99, 187-192.

Rauscher, S., Coppola, E., Piani, C., and Giorgi, F., 2010: Resolution effects on regional climate model simulations of seasonal precipitation over Europe, Clim. Dyn., 35, 685-711, doi:10.1007/s00382-009-0607-7.

Rebora, N., Molini, L., Casella, E., Comellas, A., Fiori, E., Pignone, F., Siccardi, F., Silvestro, F., Tanelli, S., and A., P., 2012: Extreme rainfall in the Mediterranean: What can we learn from observations?, J. Hydrometeorol., 14, 906-922.

Sachindra, D., Huang, F., Barton, A., and Perera, B., 2013: Statistical downscaling of general circulation model outputs to precipitation-part 2: bias-correction and future projections, Int. J. Climatol., 34, 3282-3303.

Schindelr, A., Toreti, A. and, Z. M., Scoccimarro, E., Gualdi, S., Fukutome, S., Xoplaki, E., and Luterbarcher, J., 2015: On the Internal Variability of Simulated Daily Precipitation, J. CLIMATE, 28, 3624-3630.

Scoccimarro, E., Villarini, G., Vichi, M., Zampieri, M., Fogli, P., Bellucci, A., and Gualdi, S., 2015: Projected changes in intense precipitation over Europe at the daily and sub-daily time scales, J. CLIMATE, doi:10.1175/JCLI-D-14-00779.1.

Smiatek, G., Kunstmann, H., Knoche, R., and Marx, A., 2009: Precipitation and temperature statistics in high-resolution regional climate models: Evaluation for the European Alps, J. Geophys. Res., 114, D19 107, doi:10.1029/2008JD011353.

Stocker, T. F., Qin, D., Plattner, G.-K., Tignor, M., Allen, S., Boschung, J., Nauels, A., Xia, Y., Bex, V., and P.M., M., 2013:
Contribution of Working Group I to the Fifth Assessment Report of the Intergovernmental Panel on Climate Change, Cambridge University Press, Cambridge, United Kingdom and New York, NY, USA.

Sun, F., Roderick, M., and Farquhar, G., 2012: Changes in the variability of global land precipitation, Geophys. Res. Lett., 39, L19 402, doi:10.1029/2012GL053369.

Taylor, K., Stouffer, R., and Meehl, G., 2012: An overview of CMIP5 and the experiment design, Bull. Am. Meteorol. Soc., 93, 485-498, doi:10.1175/BAMS-D-11-00094.1.

Toreti, A., Xoplaki, E., Maraun, D., Kuglitsch, F., Wanner, H., and Luterbacher, J., 2010: Characterisation of extreme winter precipitation in Mediterranean coastal sites and associated anomalous atmospheric circulation patterns., Nat. Hazards Earth Syst. Sci., 10, 1037-1050.

Trenberth, K. E., Dai, A., Rasmussen, R., and Parsons, D. B., 2003: The changing character of precipitation, Bull. Am. Meteorol. Soc., 84, 1205-1217.

Turco, M., Sanna, A., Herrera, S., Llasat, M. C., and Gutierrez, J. M., 2013: Large biases and inconsistent climate change signals in ENSEMBLES regional projections, Clim. Change, 120, 859-869.

Wilcoxon, F., 1945: Individual Comparisons by Ranking Methods, Biometrics Bulletin, 1.

WMO, 2009: Guidelines on Analysis of extremes in a changing climate in support of informed decisions for adaptation, Technical Report WCDMP No. 72, WMO/TD-No. 1500, WMO: Geneva, Switzerland. 